\title{
Improving the identification of high-z Herschel sources with position priors and optical/NIR and FIR/mm photometric redshifts ${ }^{\star}$
}

\author{
P. G. Pérez-González ${ }^{1,2}$, E. Egami ${ }^{2}$, M. Rex ${ }^{2}$, T. D. Rawle ${ }^{2}$, J.-P. Kneib ${ }^{3}$, J. Richard ${ }^{4}$, D. Johansson ${ }^{5}$, B. Altieri ${ }^{6}$, \\ A. W. Blain ${ }^{7}$, J. J. Bock ${ }^{7,8}$, F. Boone ${ }^{9}$, C. R. Bridge ${ }^{7}$, S. M. Chung ${ }^{10}$, B. Clément ${ }^{3}$, D. Clowe ${ }^{11}$, F. Combes ${ }^{12}$, \\ J.-G. Cuby ${ }^{13}$, M. Dessauges-Zavadsky ${ }^{14}$, C. D. Dowell ${ }^{7,8}$, N. Espino-Briones ${ }^{1}$, D. Fadda ${ }^{15}$, A. K. Fiedler ${ }^{2}$, \\ A. Gonzalez ${ }^{10}$, C. Horellou ${ }^{5}$, O. Ilbert ${ }^{3}$, R. J. Ivison ${ }^{16,17}$, M. Jauzac ${ }^{3}$, D. Lutz ${ }^{18}$, R. Pelló ${ }^{9}$, M. J. Pereira ${ }^{2}$, G. H. Rieke ${ }^{2}$, \\ G. Rodighiero ${ }^{19}$, D. Schaerer ${ }^{14}$, G. P. Smith ${ }^{20}$, I. Valtchanov ${ }^{6}$, G. L. Walth ${ }^{2}$, P. van der Werf ${ }^{21}$, \\ M. W. Werner ${ }^{8}$, and M. Zemcov ${ }^{7,8}$ \\ (Affiliations are available in the online edition)
}

Received 31 March 2010 / Accepted 6 May 2010

\begin{abstract}
We present preliminary results about the detection of high redshift (U)LIRGs in the Bullet cluster field by the PACS and SPIRE instruments within the Herschel Lensing Survey (HLS) Program. We describe in detail a photometric procedure designed to recover robust fluxes and deblend faint Herschel sources near the confusion noise. The method is based on the use of the positions of Spitzer/MIPS $24 \mu$ m sources as priors. Our catalogs are able to reliably $(5 \sigma)$ recover galaxies with fluxes above 6 and $10 \mathrm{mJy}$ in the PACS 100 and $160 \mu \mathrm{m}$ channels, respectively, and 12 to $18 \mathrm{mJy}$ in the SPIRE bands. We also obtain spectral energy distributions covering the optical through the far-infrared/millimeter spectral ranges of all the Herschel detected sources, and analyze them to obtain independent estimations of the photometric redshift based on either stellar population or dust emission models. We exemplify the potential of the combined use of Spitzer position priors plus independent optical and IR photometric redshifts to robustly assign optical/NIR counterparts to the sources detected by Herschel and other (sub-)mm instruments.
\end{abstract}

Key words. infrared: galaxies - submillimeter: galaxies - galaxies: evolution - galaxies: high-redshift - gravitational lensing: strong galaxies: photometry

\section{Introduction}

Based on the IRAS, ISO, and Spitzer missions, we know that luminous infrared galaxies (LIRGs) experienced significant evolution in the past $8 \mathrm{Gyr}$, roughly decreasing their typical infrared luminosity $[L($ IR) $]$ by an order of magnitude from $z \sim 1$ to $z=0$ (Flores et al. 1999; Chary \& Elbaz 2001; Pérez-González et al. 2005; Le Floc'h et al. 2005; Rodighiero et al. 2010; Magnelli et al. 2009). By $z \sim 1$, they dominated the star formation activity of the Universe, being responsible for more than $50 \%$ of the cosmic SFR density up to at least $z \sim 3$, and playing an important role in the formation of massive galaxies at $z \gtrsim 1.5$, which exhibit high star formation efficiencies (Pérez-González et al. 2008; Reddy et al. 2008; Damen et al. 2009), strong dust obscured nuclear activity (Daddi et al. 2007; Donley et al. 2008), or both. Beyond $z \sim 1$, sub-mm surveys have also detected a population of LIRGs and (mostly) ULIRGs, identified as very active stages in the formation of massive galaxies (Smail et al. 1997; Hughes et al. 1998; Chapman et al. 2003, 2005; Pope et al. 2006).

Although very useful for measuring the obscuration of optical light by interstellar dust (very abundant in the most extreme starbursts), the analysis of IR data to understand the formation and evolution of galaxies in the Universe has been classically hampered mainly by two issues. First, the sensitivity of IR telescopes has increased significantly from one mission

* Herschel is an ESA space observatory with science instruments provided by European-led Principal Investigator consortia and with important participation from NASA. to the next (and also their angular resolution, although at a lower rate), but there are still problems correctly identifying all sources detected in IR surveys, most noticeably at longer wavelengths where confusion remains severe (e.g., at 70/160 $\mu \mathrm{m}$ in the Spitzer/MIPS surveys and in the SCUBA or LABOCA sub$\mathrm{mm}$ data). Moreover, finding counterparts and getting reliable redshifts from spectroscopy for high redshift (U)LIRGs is challenging since a good fraction of them are extremely faint at optical/NIR wavelengths (Ivison et al. 2002; Wilson et al. 2008; Frayer et al. 2004). A second problem for the study of $0<z \lesssim 4$ (U)LIRGs is the availability of only one photometric data point (MIPS $24 \mu \mathrm{m}$ ) for most of them. The fits to dust emission models to calculate $L(I R)$, and from this derive a SFR (Kennicutt 1998), need a considerable extrapolation, and thus the SFR is subject to significant systematic uncertainties (Papovich et al. 2006; Daddi et al. 2007; Barro et al. 2010). In addition, the limited FIR data already available for galaxies at $z \gtrsim 2$ indicate that they have different dust properties from those observed for nearby (U)LIRGs (Chapman et al. 2005; Papovich et al. 2007; Rigby et al. 2008; Rieke et al. 2009).

The ESA Herschel Space Observatory (Pilbratt et al. 2010) alleviates these two issues. Thanks to its sensitivity and angular resolution at $100<\lambda<500 \mu \mathrm{m}$, it enables us to robustly characterize high- $z$ galaxies. In addition to the populations of IR galaxies newly discovered by Herschel, it will supply up to six photometric data points in the IR range for $0<z \lesssim 4$ galaxies detected by MIPS at $24 \mu \mathrm{m}$. These data can be used to constrain the fits to the dust emission templates. Even more novel and relevant is the combination of Herschel observations with the MIPS 
data (and also IRAC) to diminish the effects of source confusion in IR surveys. The new Herschel data indeed allow an easier and more reliable identification of counterparts from one band to the adjacent one as we move to shorter wavelengths. In addition, the Herschel bands effectively fill the gap between MIPS and (sub-)mm surveys (with SCUBA2, LABOCA, or AzTEC). By combining all these datasets, we can now use the several IR photometric data points to estimate a photometric redshift based on dust emission alone, and compare it with the estimations based on UV-to-NIR data. This can be regarded as an extension of the radio-to-IR photo- $z$ method (Carilli \& Yun 1999; Dunne et al. 2000; Rengarajan \& Takeuchi 2001; Blain et al. 2003; Aretxaga et al. 2003; Daddi et al. 2009), benefiting from a finer sampling of the emission from dust at different temperatures. The procedure can be used to improve the crosscorrelation with optical/NIR galaxy samples, or even to obtain the only redshift measurement possible for very faint (high- $z$ ) IR-bright optically-faint sources. These sources may be numerous in the Herschel surveys, because of its impressive sensitivity at wavelengths probing the peak of the dust emission, which can be brighter than the optical emission by orders of magnitude.

In this letter, we perform a preliminary analysis of the Herschel Lensing Survey (HLS, PI Egami) data taken for the Bullet cluster to show the utility of position priors and photometric redshifts obtained from UV-to-NIR and IR-to-mm data to improve the identification of Herschel and sub-mm sources with those detected at shorter wavelengths. A correct identification of IR sources will be extremely relevant to all Herschel cosmological surveys, but is of critical importance for clusters, because of their crowded nature and the possible presence of high- $z$ lensed and distorted sources with very faint optical counterparts that may even only be detectable in the FIR/mm range.

\section{Data and catalogs}

The reduction of the Herschel SPIRE (Griffin et al. 2010) and PACS (Poglitsch et al. 2010) data of the Bullet cluster $(\alpha \sim$ 06:58, $\delta \sim-55: 57$ ) is described in Egami et al. (2010) and Rex et al. (2010), jointly with optical-to-mm ancillary data used to estimate photometric redshifts, stellar masses, and SFRs. Here we describe our cataloging procedure for the five Herschel bands: PACS 100 and $160 \mu \mathrm{m}$, and SPIRE 250, 350, and $500 \mu \mathrm{m}$.

Given the remarkable depth of the Spitzer MIPS $24 \mu \mathrm{m}$ images, PACS catalogs have been compiled with a position prior technique, involving a list of MIPS sources and a PSF fitting analysis (see Pérez-González et al. 2008). To improve the reliability of the method, we first aligned all Herschel images to the WCS of the MIPS $24 \mu \mathrm{m}$ map using the wcstools software (Mink 1999), obtaining matching uncertainties smaller than one pixel for each Herschel band. The MIPS $24 \mu \mathrm{m}$ catalog in the Bullet cluster field has a $5 \sigma$ detection level of $S_{5 \sigma}$ [24] $=85 \pm$ $30 \mu \mathrm{Jy}^{1}$, and a density of 5.3 sources/ $\operatorname{arcmin}^{2}$ above that threshold (7.2 sources/arcmin ${ }^{2}$ with $S$ [24] $\left.>50 \mu \mathrm{Jy}\right)$.

The similar PSF sizes of the MIPS $24 \mu \mathrm{m}$ and the PACS $100 \mu \mathrm{m}$ bands $\left(\sim 6^{\prime \prime}\right.$ and $\sim 8^{\prime \prime}$, respectively) make the identification and extraction of sources straightforward for this band. In addition, we also considered that some sources may be too faint for MIPS yet still be detectable by PACS. To account for this population, we detected sources directly in the PACS data using Sextractor (Bertin \& Arnouts 1996), obtaining a sample of 43 sources above the $5 \sigma$ level $\left(S_{5 \sigma}[100]=5.5 \pm 0.7 \mathrm{mJy}\right)$.

\footnotetext{
1 Calculated from several artificial apertures formed with random sky pixels; see Appendix A in Pérez-González et al. (2008).
}

The cross-correlation of this sample with the MIPS catalog (using a search radius of $3^{\prime \prime}$ ) revealed that all but one of the robust PACS detections are detected at $24 \mu \mathrm{m}$, and 5 of them had 2 MIPS counterparts within the search radius. The only nondetection at $24 \mu \mathrm{m}$ is, in fact, a group of three very faint $100 \mu \mathrm{m}$ sources within $10^{\prime \prime}$, which are detected as one single source by Sextractor in the PACS image, but are individual sources at $24 \mu \mathrm{m}$. The 5 multiple detections were sources for which the slightly higher resolution and greater depth of MIPS allowed the deblending of close IR emitters. This cross-correlation also found that the WCS alignment between both images has an rms smaller than $1^{\prime \prime}$.

For the $160 \mu \mathrm{m}$ image, it is expected that more $24 \mu \mathrm{m}$ sources are merged to form single sources. For this reason, before measuring photometry, we removed sources from the main cata$\log$ that could not be separated at the resolution of the PACS $160 \mu \mathrm{m}$ band, i.e., sources closer than $6^{\prime \prime}$, which is half of the PSF FWHM, keeping only the brightest source at $24 \mu \mathrm{m}$ for each group of merged sources. The master catalog combined the MIPS source list with the direct detections in the $160 \mu \mathrm{m}$ image above the $5 \sigma$ level $\left(S_{5 \sigma}[160]=10 \pm 1 \mathrm{mJy}\right)$, all the latter being actually detected in the MIPS data. Merged sources account for almost $20 \%$ of the master catalog, with $85 \%$ (15\%) of this subsample having 2 (3) objects within 6 ". Just considering sources directly detected at $160 \mu \mathrm{m}$, the fraction of them presenting multiple identifications in the MIPS catalog is higher than $40 \%$. The WCS accuracy between the MIPS and PACS160 images is inferior to $1.5^{\prime \prime}$.

The final purged list of sources was used to fit PSFs at the given positions using the daophot package in IRAF, allowing for one pixel centering offsets. The results of this PSF fitting algorithm are flux densities in a given aperture (we found the optimum values to be $5^{\prime \prime}$ and $7.5^{\prime \prime}$ for the PACS green and red channels, respectively), to which an aperture correction must be applied. The values we applied are $1.84 \pm 0.08$ and $1.93 \pm 0.08$ for 100 and $160 \mu \mathrm{m}$, respectively. We note that these aperture corrections are larger than those published by the PACS Team, but we found that their PSF matched ours (constructed with the same Bullet cluster data) for large radii, but was brighter for similar and smaller radii than that selected for the PSF fitting method.

The SPIRE catalogs were also compiled with a method based on priors and PSF fitting. The photometry was carried out in circular apertures (of radii $12^{\prime \prime}, 18^{\prime \prime}$, and $22^{\prime \prime}$ for 250,350 , and $500 \mu \mathrm{m}$, respectively), applying a calibration based on the beam sizes (assuming they are Gaussians). For the $250 \mu \mathrm{m}$ channel, we combined the list of sources detected by MIPS at $24 \mu \mathrm{m}$ and the direct detection in the SPIRE data using Sextractor and a $5 \sigma$ threshold $\left(S_{5 \sigma}[250]=12 \pm 2 \mathrm{mJy}\right)$. Other objects within $9^{\prime \prime}$ (half of the PSF FWHM) of a given candidate source were purged, only the position of the brightest galaxy at $24 \mu \mathrm{m}$ being kept. For the region covered simultaneously by MIPS and the SPIRE $250 \mu \mathrm{m}$ channel, only 2 SPIRE sources out of 261 were not detected by MIPS, but both of them turned to be $F(24) \sim 60 \mu \mathrm{Jy}$ detections at the $3 \sigma$ level. Around half of the SPIRE sources have 2 or more MIPS counterparts within $9^{\prime \prime}$. The WCS alignment between the MIPS and SPIRE250 images has an rms of $\sim 4^{\prime \prime}$.

The catalog for the SPIRE $350 \mu \mathrm{m}$ channel was constructed from the list of detections at $250 \mu \mathrm{m}$. Sources within 14" were merged into the same object (about $40 \%$ of the total catalog, virtually all of them being a doublet), only the position of the brightest one at $250 \mu \mathrm{m}$ being kept. A direct detection in the $350 \mu \mathrm{m}$ image $\left(S_{5 \sigma}[350]=17 \pm 3 \mathrm{mJy}\right)$ found that $5 \%$ of sources are not directly linked to a $250 \mu \mathrm{m}$ emitter, but virtually all of 

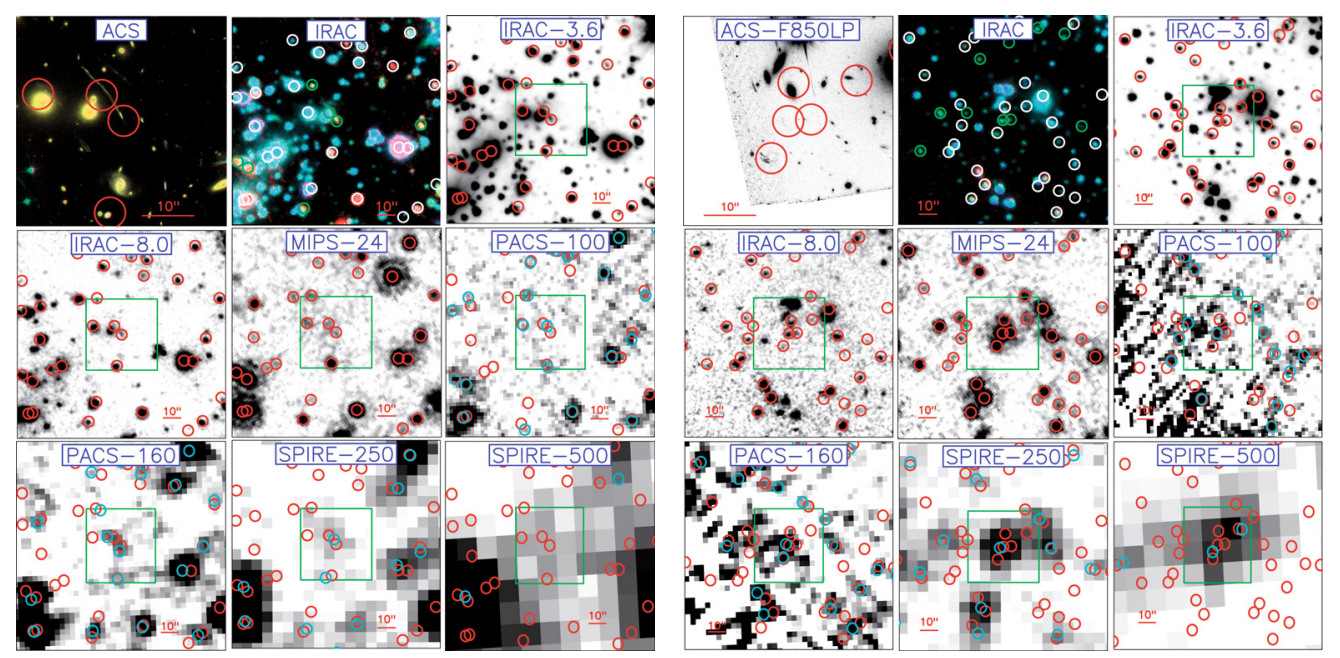

Fig. 1. Postage stamps of two high- $z$ sources in the Bullet cluster field detected by Herschel: the southern tip of the arc MIPS06:58:31.1-55:56:39.2 (left), and the LABOCA source MIPS06:58:45.3-55:58:46.5 (right). The size of the images is $2^{\prime} \times 2^{\prime}$, except for the HST images, whose size is $40^{\prime \prime} \times 40^{\prime \prime}$ (shown in the other stamps with a square). Circles (3" radius) pinpoint sources detected at $24 \mu \mathrm{m}$. Cyan circles show the actual $>5 \sigma$ detections in each Herschel band. Green circles in the IRAC RGB images mark $z>2$ galaxies in each field, including the three lensed sources in Gonzalez et al. (2009, to the SE or the arc) and a LABOCA source from Johansson et al. (2010, to the W of the arc).

Table 1. Properties of the HLS Bullet cluster catalogs.

\begin{tabular}{lccccc}
\hline \hline & PACS100 & PACS160 & SPIRE250 & SPIRE350 & SPIRE500 \\
\hline$A^{a}$ & 28.5 & 29.7 & 434 & 438 & 416 \\
$S(\lambda)^{b}$ & $5.5(3.0)$ & $10(6)$ & $12(7)$ & $17(10)$ & $18(11)$ \\
$D^{c}$ & $2.0(5.0)$ & $2.0(4.2)$ & $1.1(1.8)$ & $0.5(1.2)$ & $0.2(0.6)$ \\
$M^{d}$ & $12 \%(24)$ & $19 \%(24)$ & $48 \%(24)$ & $42 \%(250)$ & $48 \%(350)$ \\
\hline
\end{tabular}

Notes. ${ }^{(a)}$ Surveyed area in $\operatorname{arcmin}^{2} ;{ }^{(b)} 5 \sigma(3 \sigma)$ flux density detection levels ${ }^{1}$ in $\mathrm{mJy} ;{ }^{(c)}$ source densities for each significance level in sources/ $\operatorname{arcmin}^{2}$; ${ }^{(d)}$ fraction of merged sources and wavelength (in $\mu \mathrm{m}$ ) used to obtain position priors.

these are a combination of several $250 \mu \mathrm{m}$ sources that causes a displaced $350 \mu \mathrm{m}$ centroid. Only one isolated high-confidence $350 \mu \mathrm{m}$ source was not detected in the $250 \mu \mathrm{m}$ catalog, but a reliable flux could be recovered in the blue channel once the $350 \mu \mathrm{m}$ detection revealed its position. The WCS alignment between the MIPS and SPIRE350 images has an rms of $\sim 6^{\prime \prime}$.

For the $500 \mu \mathrm{m}$ channel $\left(S_{5 \sigma}[500]=18 \pm 4 \mathrm{mJy}\right)$, we started from the $350 \mu \mathrm{m}$ catalog, and we did not find any object without an identification in the bluer bands using a $18^{\prime \prime}$ search radius (approximately half of the PSF FWHM). Around 50\% of the SPIRE $500 \mu \mathrm{m}$ sources were doublets in the $350 \mu \mathrm{m}$ band. The WCS alignment between the MIPS and SPIRE500 images has an rms of $\sim 9^{\prime \prime}$.

The prior-based photometric technique was compared with the direct blind detection in the Herschel images. In PACS, a direct detection down to $5 \sigma$ included more than $40 \%$ of sources with no counterpart in the MIPS map, implying that these are probably spurious detections. The fluxes of sources extracted with both the blind and prior-based detections are virtually identical. In SPIRE, the prior-based procedure is able to recover fluxes for $30 \%$ more sources than the direct detection. For the sources in common, the average difference in the photometry is $2 \%$ (the blind method provides brighter fluxes), with a $6 \%$ scatter.

Table 1 indicates the surveyed areas, detection thresholds, the corresponding source densities, and fraction of merged sources for our catalogs of the PACS and SPIRE HLS data. An important (and potentially dangerous) step in our photometric procedure based on position priors is the blending of nearby non-resolved sources, which provides only the position of the brightest galaxy in the parent catalog to the PSF fitting algorithm. We note that the MIPS $24 \mu \mathrm{m}$ flux is dominated by the emission of warm dust and/or PAHs while the SPIRE bands are dominated by the emission of cold dust. Thus, it is probable that bright MIR emitters are not the dominant sources at FIR wavelengths (see Fig. 1). In the following section, we illustrate a method to help in the robust identification and/or deblending of the Herschel sources.

\section{Identification of high-z Herschel sources}

Figure 1 shows thumbnails in several bands of two interesting sources detected by Herschel in the Bullet cluster field: the southern tip of the arc at $z=3.24$ described in Mehlert et al. (2001), and a sub-mm galaxy detected by LABOCA (source \#10 in Johansson et al. 2010). With these two examples, we demonstrate that the use of IRAC and MIPS data helps to correctly identify the Herschel sources with optical/NIR counterparts. In the case of the arc, the photometric method based on the use of the MIPS $24 \mu \mathrm{m}$ catalog as a prior helped in the detection and measurement processes for the PACS $160 \mu \mathrm{m}$ and SPIRE bands, where the source is very faint and almost indistinguishable from the background. In the PACS red band, our method allows a deblending of the source in the two knots detected by MIPS and linked to the arc. In the case of the LABOCA source, although it is barely detected in the optical, the position of the source can be followed as we move to redder wavelengths and larger PSFs, allowing a more reliable identification in spite of the presence of a close neighbor to the west. The brightest source at $24 \mu \mathrm{m}$ is indeed already brighter than its companion at IRAC wavelengths (most noticeably, at $8.0 \mu \mathrm{m}$ ), and this nearby source disappears in PACS. This illustrates the benefits of the use of Spitzer data as priors to obtain the most probable identifications of Herschel and (sub-)mm emitters at shorter wavelengths.

Figure 2 shows the optical-to-mm SEDs of the two examples of high $-z$ galaxies. Photometric redshifts for all $24 \mu \mathrm{m}$ sources in the Bullet cluster field were estimated from the UVto-MIR data (including IRAC fluxes) using the technique described in Pérez-González et al. (2005, 2008). Briefly, the SEDs compiled from aperture-matched photometry are fitted with stellar population models and AGN templates from Polletta et al. (2007). A minimization algorithm is used to search for the optimal redshifted template fitting the data, and the most probable 
A\&A 518, L15 (2010)

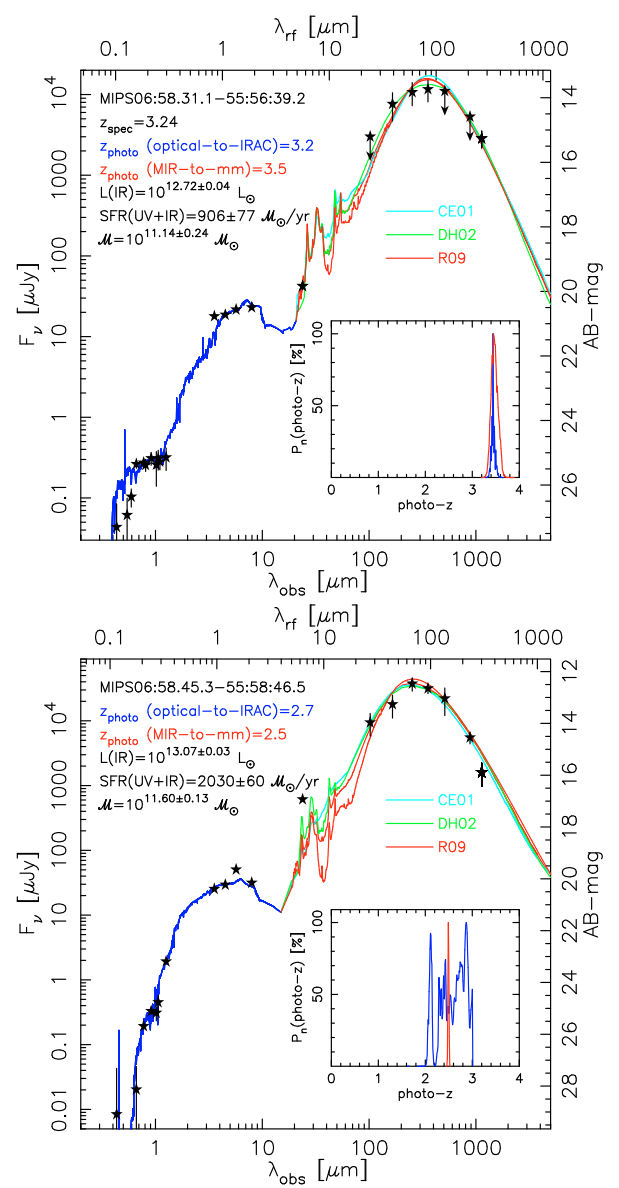

Fig. 2. SEDs of the $z=3.24$ LIRG MIPS06:58:31.1-55:56:39.2 (magnification factor $\sim 21$ ) and the $z \sim 2.5$ HyLIRG MIPS06:58:45.355:58:46.5, detected in the MIR/FIR by Spitzer and Herschel, and the (sub-)mm by LABOCA and AzTEC. The blue line shows the best-fit stellar population model for the photometry up to $8 \mu \mathrm{m}$, which provides an estimate of the photometric redshift and the stellar mass. The cyan, green, and red lines show the best-fit dust emission model for the MIRto-mm photometry (from Chary \& Elbaz 2001; Dale \& Helou 2002; and Rieke et al. 2009), which gives an independent photometric redshift estimation and a SFR value (given in the plots with no correction for magnification factor). The inset plots depict the photometric redshift normalized probability distributions for the optical/NIR and the $\mathrm{MIR} / \mathrm{mm}$ estimations.

photometric redshift is calculated by integrating the redshift probability function (see also Barro et al. 2010). Photometric redshifts were also estimated from the IR data alone by comparing the Spitzer, Herschel and (sub-)mm fluxes with dust emission templates from Chary \& Elbaz (2001), Dale \& Helou (2002), and Rieke et al. (2009). Using these two fits, we estimated relevant parameters such as the stellar mass and the SFR. The Rieke et al. (2009) template most closely fitting the data for the arc corresponds to a local $L($ IR $)=10^{11.5} L_{\odot}$ galaxy. This value is consistent with the average for the arc based on the three template libraries, $L(\mathrm{IR})=10^{11.40 \pm 0.04} L_{\odot}$, once a magnification factor of $\sim 21$ is applied (Bradač et al. 2006). The LABOCA source qualifies as a hyper-LIRG based on the best fits to all template libraries (if it is not lensed), and the best-fit template from Rieke et al. is that of a local galaxy with $10^{12.75} L_{\odot}$, although our source seems to have more prominent PAH emission.

Figure 2 illustrates the power of using independent estimates of the photometric redshift based on UV-to-NIR data and IR data alone to validate the individual values (see the photo- $z$ probability distributions). The procedure is also useful for achieving more reliable identifications of the IR emitters. For example, for the LABOCA source at $z \sim 2.5$, we calculated photometric redshifts based on UV-to-NIR data for the three close neighbors detected by MIPS within $\sim 10^{\prime \prime}$ of the central source. The galaxy to the north lies at $z \sim 0.3$ (a cluster member), that to the south at $z \sim 1.9$, and the closest companion has a photo- $z$ compatible with the central source $(z \sim 2.3 \pm 0.3)$, thus implying that the two sources are a possible interacting pair.

The general validity of our method for the whole sample of Herschel sources will be tested thoroughly in forthcoming papers by evaluating the quality of our photo-z's. From a preliminary comparison of the optical and FIR photo-z's for sources detected by MIPS, PACS, SPIRE, and a (sub)-mm instrument, it is encouraging that we measure an average $\Delta(z) /(1+z)=\eta=0.09$ with $10 \%$ of catastrophic outliers $(\eta>0.2)$, comparable to the typical goodness of IR-based photo-z's (Aretxaga et al. 2003).

Acknowledgements. This work is based in part on observations made with Spitzer, operated by JPL/Caltech. We thank the AzTEC Team for letting us use their data. P.G.P.-G. acknowledges support from grants AYA 2006-02358, AYA 2006-15698-C02-02, and CSD2006-00070, and the Ramón y Cajal Program, all financed by the Spanish Government and/or the European Union.

\section{References}

Aretxaga, I., Hughes, D. H., Chapin, E. L., et al. 2003, MNRAS, 342, 759 Barro, G., Pérez-González, P. G., Gallego, J., et al. 2010, ApJS, submitted Bertin, E., \& Arnouts, S. 1996, A\&AS, 117, 393

Blain, A. W., Barnard, V. E., \& Chapman, S. C. 2003, MNRAS, 338, 733

Bradač, M., Clowe, D., Gonzalez, A. H., et al. 2006, ApJ, 652, 937

Carilli, C. L., \& Yun, M. S. 1999, ApJ, 513, L13

Chapman, S. C., et al. 2003, Nature, 422, 695

Chapman, S. C., Blain, A. W., Smail, I., \& Ivison, R. J. 2005, ApJ, 622, 772

Chary, R., \& Elbaz, D. 2001, ApJ, 556, 562

Daddi, E., Alexander, D. M., Dickinson, M., et al. 2007, ApJ, 670, 173

Daddi, E., Dannerbauer, H., Stern, D., et al. 2009, ApJ, 694, 1517

Dale, D. A., \& Helou, G. 2002, ApJ, 576, 159

Damen, M., Förster Schreiber, N. M., Franx, M., et al. 2009, ApJ, 705, 617

Donley, J. L., et al. 2008, ApJ, 687, 111

Dunne, L., Clements, D. L., \& Eales, S. A. 2000, MNRAS, 319, 813

Egami, E., et al. 2010, A\&A, 518, L12

Flores, H., Hammer, F., Thuan, T. X., et al. 1999, ApJ, 517, 148

Frayer, D. T., Chapman, S. C., Yan, L., et al. 2004, ApJS, 154, 137

Gonzalez, A. H., Clowe, D., Bradač, M., et al. 2009, ApJ, 691, 525

Griffin, M. J., et al. 2010, A\&A, 518, L3

Hughes, D. H., Serjeant, S., Dunlop, J., et al. 1998, Nature, 394, 241

Ivison, R. J., Greve, T. R., Smail, I., et al. 2002, MNRAS, 337, 1

Johansson, D., Horellou, C., et al. 2010, A\&A, 514, A77

Kennicutt, R. C. 1998, ARA\&A, 36, 189

Le Floc'h, E., Papovich, C., Dole, H., et al. 2005, ApJ, 632, 169

Magnelli, B., Elbaz, D., Chary, R. R., et al. 2009, A\&A, 496, 57

Mehlert, D., Seitz, S., Saglia, R. P., et al. 2001, A\&A, 379, 96

Mink, D. J. 1999, in Astronomical Data Analysis Software and Systems VIII, ed. D. M. Mehringer, et al. ASP Conf. Ser., 172, 498

Pérez-González, P. G., Rieke, G. H., Egami, E., et al. 2005, ApJ, 630, 82 Pérez-González, P. G., Rieke, G. H., Villar, V., et al. 2008, ApJ, 675, 234 Papovich, C., Moustakas, L. A., Dickinson, M., et al. 2006, ApJ, 640, 92 Papovich, C., Rudnick, G., Le Floc'h, E., et al. 2007, ApJ, 668, 45 Pilbratt, G. L., et al. 2010, A\&A, 518, L1

Poglitsch, A., et al. 2010, A\&A, 518, L2

Polletta, M., Tajer, M., Maraschi, L., et al. 2007, ApJ, 663, 81

Pope, A., Scott, D., Dickinson, M., et al. 2006, MNRAS, 370, 1185

Reddy, N. A., Steidel, C. C., Pettini, M., et al. 2008, ApJS, 175, 48

Rengarajan, T. N., \& Takeuchi, T. T. 2001, PASJ, 53, 433

Rex, M., et al. 2010, A\&A, 518, L13

Rieke, G. H., Alonso-Herrero, A., Weiner, B. J., et al. 2009, ApJ, 692, 556 Rigby, J. R., Marcillac, D., Egami, E., et al. 2008, ApJ, 675, 262

Rodighiero, G., Vaccari, M., Franceschini, A., et al. 2010, A\&A, 515, A8

Smail, I., Ivison, R. J., \& Blain, A. W. 1997, ApJ, 490, L5

Wilson, G. W., Hughes, D. H., Aretxaga, I., et al. 2008, MNRAS, 390, 1061

Page 5 is available in the electronic edition of the journal at http://www . aanda . org 
1 Departamento de Astrofísica, Facultad de CC. Físicas, Universidad Complutense de Madrid, 28040 Madrid, Spain

e-mail: pgperez@astrax.fis.ucm.es

2 Steward Observatory, The University of Arizona, $933 \mathrm{~N}$ Cherry Avenue, Tucson, AZ 85721, USA

${ }^{3}$ Laboratoire d'Astrophysique de Marseille, CNRS - Université AixMarseille, 38 Rue Frédéric Joliot-Curie, 13388 Marseille, France

${ }^{4}$ Institute for Computational Cosmology, Department of Physics, Durham University, South Road, Durham DH1 3LE, UK

5 Onsala Space Observatory, Chalmers University of Technology, 439 92 Onsala, Sweden

${ }^{6}$ Herschel Science Centre, ESAC, ESA, PO Box 50727, 28080 Madrid, Spain

7 California Institute of Technology, Pasadena, CA 91125, USA

8 Jet Propulsion Laboratory, Pasadena, CA 91109, USA

9 Laboratoire d'Astrophysique de Toulouse-Tarbes, Université de Toulouse, CNRS, 14 Av. Edouard Belin, 31400 Toulouse, France

10 Department of Astronomy, University of Florida, Gainesville, FL 32611-2055, USA

11 Department of Physics \& Astronomy, Ohio University, Clippinger Labs 251B, Athens, OH 45701, USA
12 Observatoire de Paris, LERMA, 61 Av. de l'Observatoire, 75014 Paris, France

13 Laboratoire d'Astrophysique de Marseille, Observatoire Astronomique de Marseille-Provence, 2 Place Le Verrier, 13248 Marseille, France

14 Geneva Observatory, University of Geneva, $51 \mathrm{Ch}$. des Maillettes, 1290 Versoix, Switzerland

15 NASA Herschel Science Center, California Institute of Technology, MS 100-22, Pasadena, CA 91125, USA

16 UK Astronomy Technology Centre, Science and Technology Facilities Council, Royal Observatory, Blackford Hill, Edinburgh EH9 3HJ, UK

17 Institute for Astronomy, University of Edinburgh, Blackford Hill, Edinburgh EH9 3HJ, UK

18 Max-Planck-Institut für extraterrestrische Physik, Postfach 1312, 85741 Garching, Germany

19 Department of Astronomy, University of Padova, Vicolo dell'Osservatorio 3, 35122 Padova, Italy

20 School of Physics and Astronomy, University of Birmingham, Edgbaston, Birmingham, B15 2TT, UK

21 Sterrewacht Leiden, Leiden University, PO Box 9513, 2300 RA Leiden, The Netherlands 